\title{
Methyltransferases excised from trans-AT polyketide synthases operate on $\mathrm{N}$-acetylcysteamine-bound substrates
}

\author{
D Cole Stevens ${ }^{1,3}$, Drew T Wagner ${ }^{1,3}$, Hannah R Manion ${ }^{1}$, Bradley K Alexander ${ }^{1}$ and \\ Adrian T Keatinge-Clay ${ }^{1,2}$
}

The Journal of Antibiotics (2016) 69, 567-570; doi:10.1038/ja.2016.66; published online 15 June 2016

\begin{abstract}
INTRODUCTION
Trans-acyltransferase polyketide synthases (trans-AT PKSs) construct biologically active polyketides, such as the antibiotic mupirocin, in an assembly line process catalyzed by collections of enzymatic domains termed modules. Each module of a trans-AT possesses an acyl carrier protein (ACP) and ketosynthase (KS) that, along with a separately-encoded acyltransferase (AT) domain, constitute the minimal trio of domains required for the extension of a covalently-bound intermediate. ${ }^{1,2}$ Further modifications at the $\alpha$ - and $\beta$-carbons of the polyketide are introduced by processing enzymes such as the methyltransferase (MT), ketoreductase (KR), dehydratase (DH) and enoylreductase (ER), depending on their inclusion within a module. This optional incorporation can be primarily credited for the vast chemical and functional diversity observed within polyketides. ${ }^{1,2}$

Unlike the embedded AT domains of cis-AT PKS pathways that deliver malonyl-CoA or methylmalonyl-CoA extender units to a single module, the discrete ATs of trans-AT PKSs deliver extender units to several modules. Since these ATs are primarily selective for malonyl groups, trans-AT PKSs employ embedded S-adenosyl methionine (SAM)-dependent MT domains to install methyl groups into polyketide backbones. ${ }^{1-3}$ Although SAM-dependent MTs constitute a well-studied superfamily of enzymes and are relatively common within trans-AT PKSs, a dearth of information exists for trans-AT MTs and PKS-embedded MTs in general. Only a few studies of MTs from select cis-AT PKSs and fungal highly reducing PKSs (HR-PKSs) have been reported. ${ }^{4-9}$ Analysis of a fungal HR-PKS responsible for the production of lovastatin revealed the kinetic preference of an embedded MT towards its native acyl-S-N-acetylcysteamine (NAC) substrate analog. $1,2,9,10,11$
\end{abstract}

To explore the selectivity of trans-AT MTs, we examined the methylation activity of excised MT domains from three well-known trans-AT pathways, responsible for the production of the antimicrobial agents bacillaene, difficidin and mupirocin (Figure 1), toward the acyl-S-NAC substrates 3-oxobutanoyl$S$-NAC (1), 3-oxopentanoyl-S-NAC (2) and 3-oxohexanoyl$S$-NAC (3). ${ }^{12-14}$ (Figure 2a). This is the first account of the substrate specificity and activity of excised MT domains from trans-AT PKSs.

\section{MATERIALS AND METHODS}

$S$-adenosyl-methionine (Ark Pharm, Inc, Libertyville, IL, USA) was dissolved in $300 \mathrm{~mm}$ sodium phosphate buffer ( $\mathrm{pH} \mathrm{7.8)} \mathrm{to} \mathrm{a} \mathrm{final}$ concentration of $150 \mathrm{~mm}$.

The DNA encoding DifMT1, DifMT6, DifMT13, BaeMT9, BaeMT14, MupMT1 and MupMT3 was amplified from the difficidin and bacillaene gene clusters of Bacillus amyloliquefaciens FZB42 and the mupirocin gene cluster of Pseudomonas fluorescens 13525 and inserted into pGAY28b, a ligation-independent cloning vector constructed from pET28b (Supplementary Table S1). ${ }^{15}$ Escherichia coli BL21(DE3) transformed with the expression plasmid was inoculated into $\mathrm{LB}$ media containing $50 \mathrm{mg} \mathrm{l^{-1 }}$ kanamycin at $37^{\circ} \mathrm{C}$, grown to $\mathrm{OD}_{600}=0.5$, and induced with $0.5 \mathrm{~mm}$ isopropyl $\beta$-D-1-thiogalactopyranoside (IPTG). After $18 \mathrm{~h}$ at $15^{\circ} \mathrm{C}$, cells were collected by centrifugation and resuspended in lysis buffer $(0.5 \mathrm{M} \mathrm{NaCl}, 10 \%$ (v/ v) glycerol, $0.1 \mathrm{~m}$ HEPES, pH 7.5). Following sonication, cell debris was removed by centrifugation $(30000 \mathrm{~g}, 30 \mathrm{~min})$. The supernatant was poured over a column of Nickel-NTA resin (Thermo Scientific, Waltham, MA, USA), which was then washed with $40 \mathrm{ml}$ lysis buffer containing $15 \mathrm{~mm}$ imidazole and eluted with $5 \mathrm{ml}$ lysis buffer containing $150 \mathrm{~mm}$ imidazole. The eluted protein was concentrated to $\sim 10 \mathrm{mg} \mathrm{ml}^{-1}$ in the equilibration buffer and stored at $-80^{\circ} \mathrm{C}$ until needed. The $S$-adenosyl homocysteine (SAH) nucleosidase Pfs was amplified from E. coli BL21(DE3) genomic DNA and cloned and

\footnotetext{
${ }^{1}$ Department of Molecular Biosciences, The University of Texas at Austin, Austin, TX, USA and ${ }^{2}$ Department of Chemistry, The University of Texas at Austin, Austin, TX, USA ${ }^{3}$ These authors contributed equally to this work.

Correspondence: Professor AT Keatinge-Clay, Departments of Chemistry and Molecular Biosciences, The University of Texas at Austin, 1 University Station A5300, Austin, TX 78712, USA.

E-mail: adriankc@utexas.edu

Received 29 February 2016; revised 2 May 2016; accepted 8 May 2016; published online 15 June 2016
} 
a

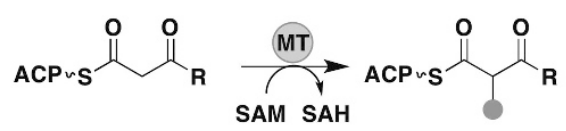

b
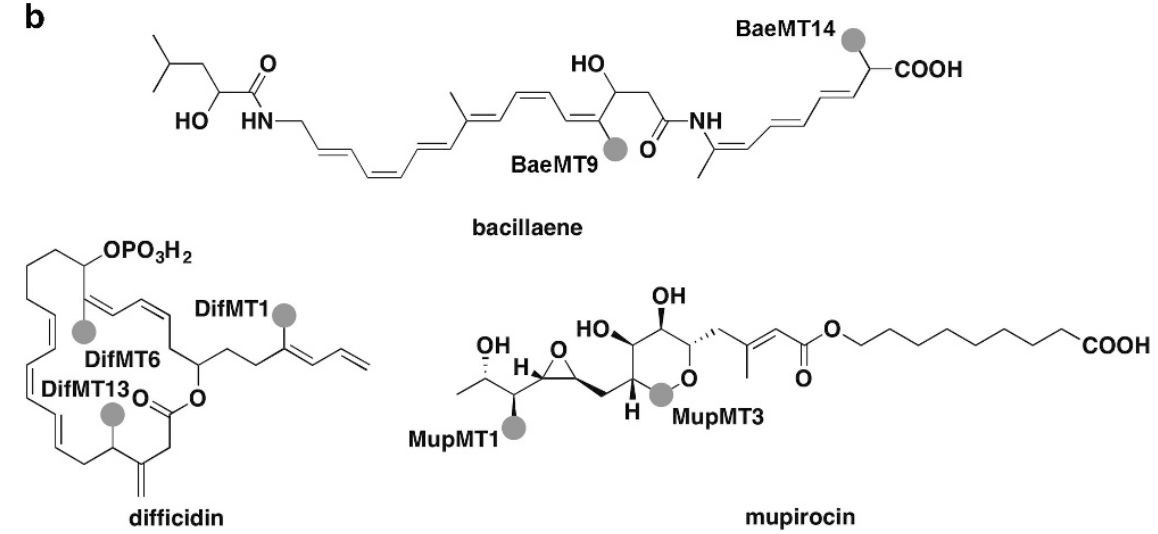

Figure 1 (a) Canonical $\alpha$-methylation catalyzed by trans-AT PKS MTs. (b) $\alpha$-branches and cognate MT domains of bacillaene, difficidin, and mupirocin. AT, acyltransferase; MT, methyltransferase; PKS, polyketide synthase. A full color version of this figure is available at The Journal of Antibiotics journal online.

purified as above (Supplementary Table S1). Reactions were supplemented with Pfs due to the potent inhibition of MTs by SAH. ${ }^{5}$

Compounds 1-3, 2-methyl-3-oxobutanoyl-S-NAC (4), and 3-oxohexanoyl-S-NAC (6) were prepared according to reported protocols, ${ }^{16}$ as was 2-methyl-3-oxopentanoyl-S-NAC (5). ${ }^{17}$

To determine MT activity reactions with substrates 1-3 (10 mm) contained $150 \mathrm{~mm}$ Tris- $\mathrm{HCl}, 100 \mathrm{~mm} \mathrm{NaCl}, 20 \mathrm{~mm}$ SAM, $10 \%(\mathrm{v} / \mathrm{v})$ glycerol, $20 \mu \mathrm{M} \mathrm{Pfs}$, and $10 \mu \mathrm{M} \mathrm{MT}$ in a total volume of $200 \mu$ l. Reactions were run in parallel at $25^{\circ} \mathrm{C}$ for $\sim 16 \mathrm{~h}$. Standards of 4, 5, and $\mathbf{6}$ at concentrations from $250 \mu \mathrm{M}$ to $50 \mathrm{~mm}$ were added directly to injection solvent and HPLC peak areas were collected to provide standard curves for observable product formation.

To determine the linear region of MT kinetic data, reactions with substrates 1-3 (10 mm) contained $150 \mathrm{~mm}$ Tris- $\mathrm{HCl}, 100 \mathrm{~mm} \mathrm{NaCl}$, $20 \mathrm{~mm}$ SAM, 10\% (v/v) glycerol, $20 \mu \mathrm{M}$ Pfs and $10 \mu \mathrm{M}$ MT in a total volume of $200 \mu \mathrm{l}$ and were run in parallel at $25^{\circ} \mathrm{C}$ and quenched at 0 , $1,2,3,4$ and 5 h.

Reactions with substrates 1-3 (100 $\mu \mathrm{M}, 500 \mu \mathrm{M}, 1 \mu \mathrm{M}, 2.5 \mathrm{~mm}, 5 \mathrm{~mm}$, $10 \mathrm{~mm}$ or $50 \mathrm{~mm}$ ) contained $150 \mathrm{~mm}$ Tris- $\mathrm{HCl}, 100 \mathrm{~mm} \mathrm{NaCl}, 20 \mathrm{~mm}$ SAM, $10 \%(\mathrm{v} / \mathrm{v})$ glycerol, $20 \mu \mathrm{M} \mathrm{Pfs}$, and $10 \mu \mathrm{M}$ MT in a total volume of $200 \mu \mathrm{l}$. Reactions were run in parallel at $25^{\circ} \mathrm{C}$ over a period of 1-3 h (MupMT1+1, 1 h; MupMT1+2, 2 h; MupMT1+3, 3 h; BaeMT9 +3, 2 h; DifMT1+2, 2 h; DifMT1+3, 2 h) (Figures 2b-g). After completion, reactions were extracted with $1 \mathrm{ml}$ of ethyl acetate. The organic layer was dried in vacuo and resuspended in methanol for HPLC analysis. Standard curves were generated by HPLC analysis of $60 \mu \mathrm{M}, 300 \mu \mathrm{M}, 600 \mu \mathrm{M}, 1 \mu \mathrm{M}, 3 \mathrm{~mm}$ and $6 \mathrm{~mm}$ injections of 4 and $200 \mu \mathrm{M}, 1 \mu \mathrm{M}, 2 \mathrm{~mm}, 5 \mathrm{~mm}, 10 \mathrm{~mm}$ and $20 \mathrm{~mm}$ injections of 5 and $\mathbf{6}$. Reactions for each set of substrate concentrations were performed in triplicate.

Product formation was quantified by absorbance with reverse phase HPLC resolution of reaction mixtures stopped at a fixed time-point with $0.1-50 \mathrm{~mm}$ substrate as well as full progress curves at $10 \mathrm{~mm}$ substrate (Supplementary Figure S10). Global fitting of both data sets to a reaction model using Kintek Explorer allowed determination of $\mathrm{k}_{\text {cat }} / \mathrm{K}_{\mathrm{M}}$ values for each enzyme/substrate pair (Table 1 and Supplementary Figure S1) ${ }^{18-20}$.
HPLC analysis was performed with a tandem Waters 2707 autosampler and Waters 1525 binary HPLC pump (Milford, MA, USA) connected to a Waters 2998 photodiode array detector using a Varian Microsorb-MV $\mathrm{C}_{18}$ column $(250 \times 4.6 \mathrm{~mm}, 5 \mu \mathrm{m}$ particle size, $100 \AA$ pore size) and mobile phases consisting of water with $0.1 \%$ TFA (solvent A) and acetonitrile with $0.1 \%$ TFA (solvent B) with a solvent gradient of $5-100 \% \mathrm{~B}$ over $30 \mathrm{~min}$ at a flow rate of $1 \mathrm{ml} \mathrm{min}^{-1}$. Highresolution MS measurements of methylated products were obtained by chemical ionization (ESI) with a VG analytical ZAB2-E instrument (Milford, MA, USA). Peak integrations were automated with a signalto-noise cutoff of $5 \%$ peak area.

\section{RESULTS AND DISCUSSION}

Of the seven domains cloned, all produced soluble protein with the exception of MupMT3. In addition to several previously identified sequence motifs that distinguish between cis- and trans-AT MT domains, a trans-AT MT sequence alignment revealed an additional notable difference between these two classes of MT domain regarding their position within the module. ${ }^{21}$ In contrast to their cis-AT counterparts, trans-AT MTs are not embedded within the KR structural subdomain but are instead usually situated immediately after the KR domain and immediately before the ACP domain. ${ }^{22}$

In vitro assays of the excised MTs BaeMT9, DifMT1, and MupMT1 showed each to be capable of catalyzing the methylation of substrates 1, 2, and 3 to afford 4, 5, and 6 (Figure 2 and Supplementary Figure S2, Table 1). These results show that, at least when separated from their assembly lines, MTs are not highly substrate-specific. Other excised PKS enzymes (KRs, DHs, ERs and thioesterases (TEs)) also demonstrate broad substrate specificities. ${ }^{20-25}$ The low reaction rates of BaeMT9, DifMT1, and MupMT1 are suggestive that local substrate concentrations within the intact assembly line are required for optimal catalytic rates. While the conversions of 1 to 4 catalyzed by BaeMT9 and DifMT1 as well as $\mathbf{2}$ to $\mathbf{5}$ catalyzed by BaeMT9 were observed overnight, the percent conversions within the linear region $(1-3 \mathrm{~h})$ were too low to accurately report kinetic parameters using HPLC analysis (Supplementary Figures S5). The observed reaction rates for MTs are lower than those of other excised processing domains 
a
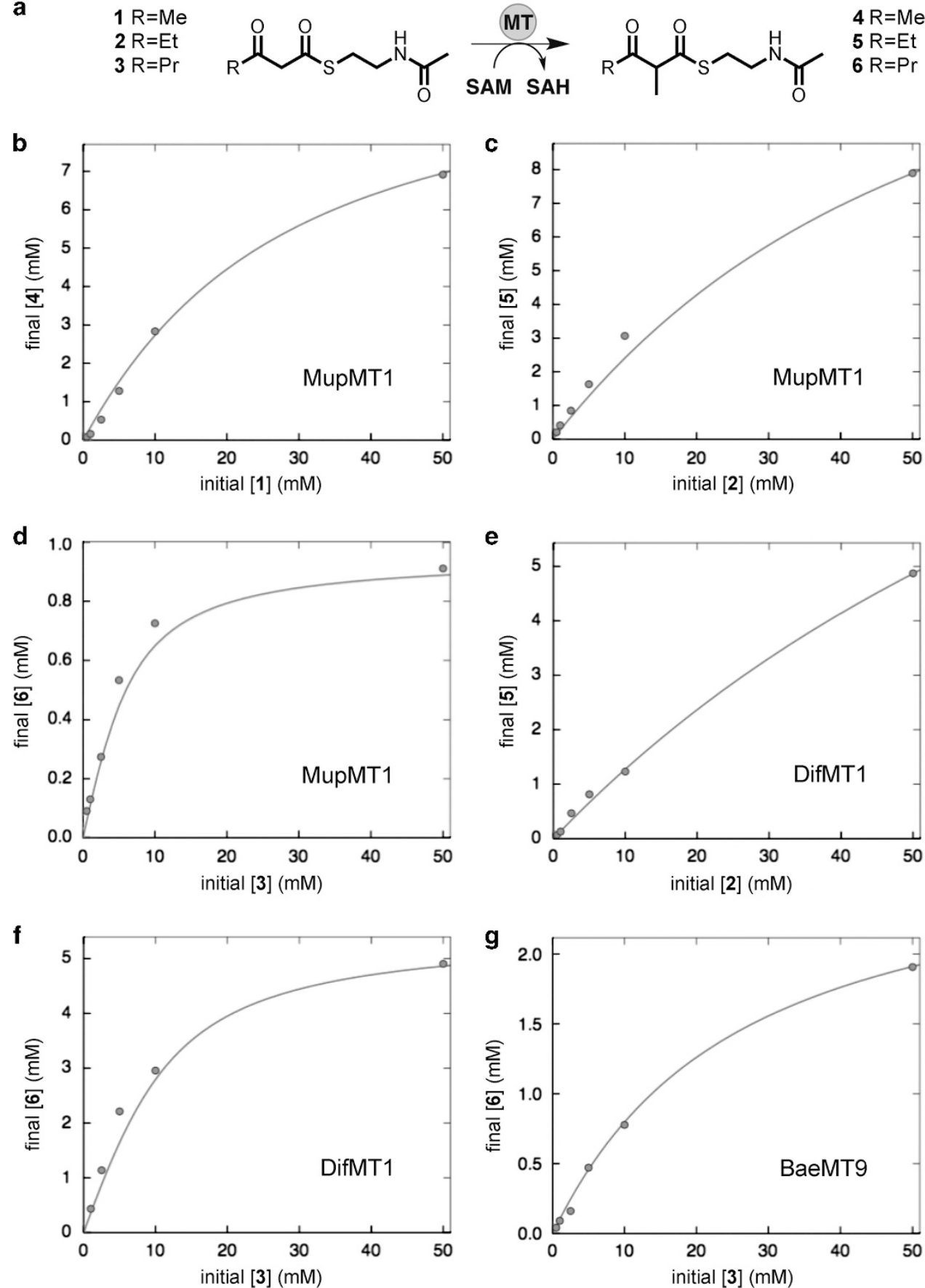

Figure 2 Saturation curves for (a) Substrates and products of MT reactions in this study, (b) MupMT1 and 1 after $1 \mathrm{~h}$, (c) MupMT1 and 2 after $2 \mathrm{~h}$, (d) MupMT1 and $\mathbf{3}$ after $3 \mathrm{~h},(\mathrm{e})$ BaeMT9 and $\mathbf{3}$ after $2 \mathrm{~h}$, (f) DifMT1 and $\mathbf{2}$ after $2 \mathrm{~h}$, (g) DifMT1 and $\mathbf{3}$ after 2 h. Experimental data from saturation curves was globally fit to a fixed concentration time-course for enzyme-substrate pairs (Supplementary Figure S1). A full color version of this figure is available at The Journal of Antibiotics journal online.

(for example, KRs, DHs or TEs) toward NAC-linked substrates. ${ }^{23-28}$ Although DifMT1 and BaeMT9 demonstrated substrate affinities for 2 and 3, respectively (Table 1), a significant kinetic preference for a specific substrate was not observed from any of the investigated MTs.

The N-terminal MTs DifMT6, DifMT13, and BaeMT14 did not catalyze a detectable amount of methylation of 1, 2 or 3 . Unlike the completely embedded MTs, N-terminal MTs may require additional components for activity, such as inclusion in their native polypeptide or interaction with the upstream PKS polypeptide.

The presented work demonstrates that, similar to the other processing domains excised from PKSs, excised trans-AT PKS MTs are capable of operating on thioester-bound polyketide substrates in vitro. These data disclose the initial investigation of the heretofore unexplored MTs found throughout the trans-AT PKS landscape and provide insight into the catalytic activity of the 
Table 1 Kinetic analysis of DifMT1, MupMT1, and BaeMT9 toward $\mathrm{N}$-acetylcysteamine substrates

\begin{tabular}{lcccc}
\hline MT & substrate & $k_{\text {cat }}\left(\mathrm{min}^{-1}\right)$ & $K_{M}(\mathrm{~mm})$ & $\begin{array}{c}k_{\text {cat }} / K_{M} \\
\left(\mathrm{~mm}^{-1} \mathrm{~min}^{-1}\right)\end{array}$ \\
\hline MupMT1 & $\mathbf{1}$ & $26 \pm 3.7$ & $22 \pm 7$ & $1.2 \pm 0.3$ \\
MupMT1 & $\mathbf{2}$ & $47 \pm 14$ & $48 \pm 24$ & $1.0 \pm 0.4$ \\
MupMT1 & $\mathbf{3}$ & $4.6 \pm 1.6$ & $3.2 \pm 3.2$ & $1.5 \pm 1.3$ \\
DifMT1 & $\mathbf{2}$ & $13 \pm 5.4$ & $110 \pm 83$ & $0.12 \pm 0.08$ \\
DifMT1 & $\mathbf{3}$ & $12 \pm 1.9$ & $5.4 \pm 3.0$ & $2.2 \pm 1.1$ \\
BaeMT9 & $\mathbf{3}$ & $5.6 \pm 2.8$ & $22 \pm 19$ & $0.25 \pm 0.17$ \\
\hline
\end{tabular}

enzyme when removed from its native module. The ability of these MTs to install methyl branches on the carbon chains of small molecule substrates makes them particularly attractive as biocatalysts.

\section{CONFLICT OF INTEREST}

The authors declare no conflict of interest.

\section{ACKNOWLEDGEMENTS}

We thank Dr Kenneth Johnson for helpful discussion pertaining to the fitting of kinetic data. We thank the University of Texas at Austin mass spectrometry facility for their help in obtaining high-resolution masses for 1-6. We thank the National Institutes of Health, National Institute of General Medical Sciences (GM106112) and the Welch Foundation (F-1712) for funding.

Author contributions: All authors have given approval to the final version of the manuscript.

1 Piel, J. Biosynthesis of polyketides by trans-AT polyketide synthases. Nat. Prod. Rep. 27, 996-1047 (2010).

2 Helfrich, E. J. \& Piel, J. Biosynthesis of polyketides by trans-AT polyketide synthases. Nat. Prod. Rep. 33, 231-316 (2016).

3 Keatinge-Clay, A. T. The structures of type I polyketide synthases. Nat. Prod. Rep. 29, 1050-1073 (2012).

4 Ansari, M. Z., Sharma, J., Gokhale, S. \& Mohanty, D. In silico analysis of methyltransferase domains involved in biosynthesis of secondary metabolites. BMC Bioinformatics 9, 454 (2008).

5 Liscombe, D. K., Louie, G. V. \& Noel, J. P. Architectures, mechanisms and molecular evolution of natural product methyltransferases. Nat. Prod. Rep. 29, $1238-1250$ (2012)

6 Miller, D. A., Luo, L., Hillson, N., Keating, T. A. \& Walsh, C. T. Yersiniabactin synthetase: a four-protein assembly line producing the nonribosomal peptide/ polyketide hybrid siderophore of Yersinia pestis. Chem. Biol. 9, 333-344 (2002)

7 Poust, S. et al. Divergent mechanistic routes for the formation of gem-dimethyl groups in the biosynthesis of complex polyketides. Angew. Chem. Int. Ed. 54, 2370 (2015).
8 Winter, J.M. et al. Expanding the structural diversity of polyketides by exploring the cofactor tolerance of an inline methyltransferase domain. Org. Lett. 15, 3775-3777 (2013)

9 Cacho, R. A. et al. Understanding Programming of Fungal Iterative Polyketide Synthases: The Biochemical Basis for Regioselectivity by the Methyltransferase Domain in the Lovastatin Megasynthase. J. Am. Chem. Soc. 137, 15688-15691 (2015).

$10 \mathrm{Jenner,} \mathrm{M.} \mathrm{et} \mathrm{al.} \mathrm{Substrate} \mathrm{specificity} \mathrm{in} \mathrm{ketosynthase} \mathrm{domains} \mathrm{from} \mathrm{trans-AT} \mathrm{polyketide}$ synthases. Angew. Chem. Int. Ed. 52, 1143-1147 (2013).

11 Jenner, M. et al. Acyl-chain elongation drives ketosynthase substrate selectivity in transacyltransferase polyketide synthases. Angew. Chem. Int. Ed. 54, 1817-1821 (2015).

12 Moldenhauer, J. et al. The final steps of bacillaene biosynthesis in Bacillus amyloliquefaciens FZB42: direct evidence for beta,gamma dehydration by a trans-acyltransferase polyketide synthase. Angew. Chem. Int. Ed. 49, 1465-1467 (2010).

13 Chen, X.H. et al. Structural and functional characterization of three polyketide synthase gene clusters in Bacillus amyloliquefaciens FZB 42. J. Bacteriol. 188, 4024-4036 (2006)

14 Gurney, R. \& Thomas, C. M. Mupirocin: biosynthesis, special features and applications of an antibiotic from a gram-negative bacterium. Appl. Microbiol. Biotechnol. 90, 11-21 (2011).

15 Gay, G., Wagner, D. T., Keatinge-Clay, A. T. \& Gay, D. C. Rapid modification of the pET-28 expression vector for ligation independent cloning using homologous recombination in Saccharomyces cerevisiae. Plasmid 7, 66-71 (2014).

16 Piasecki, S. K. et al. Employing modular polyketide synthase ketoreductases as biocatalysts in the preparative chemoenzymatic syntheses of diketide chiral building blocks. Chem. Biol. 18, 1331-1340 (2011).

17 Bailey, C. B., Pasman, M. E. \& Keatinge-Clay, A. T. Substrate structure-activity relationships guide rational engineering of modular polyketide synthase ketoreductases. Chem. Commun. (Camb) 52, 792-795 (2016).

18 Johnson, K. A., Simpson, Z. B. \& Blom, T. Global Kinetic Explorer: A new computer program for dynamic simulation and fitting of kinetic data. Anal. Biochem. 387, 20-29 (2009).

19 Johnson, K. A., Simpson, Z. B. \& Blom, T. FitSpace Explorer: An algorithm to evaluate multi-dimensional parameter space in fitting kinetic data. Anal. Biochem. 387, 30-41 (2009).

20 Johnson, K. A. Fitting enzyme kinetic data with KinTek global kinetic explorer. Methods Enzymol. 467, 601-626 (2009).

21 Young, J. et al. Elucidation of gephyronic acid biosynthetic pathway revealed unexpected SAM-dependent methylations. J. Nat. Prod. 76, 2269-2276 (2013).

22 Nguyen, T. et al. Exploiting the mosaic structure of trans-acyltransferase polyketide synthases for natural product discovery and pathway dissection. Nat. Biotechnol. 26, 225-233 (2008).

23 Siskos, A. P. et al. Molecular basis of Celmer's rules: stereochemistry of catalysis by isolated ketoreductase domains from modular polyketide synthases. Chem. Biol. 12, 1145-1153 (2005).

$24 \mathrm{Li}, \mathrm{Y}$. et al. Polyketide intermediate mimics as probes for revealing cryptic stereochemistry of ketoreductase domains. ACS Chem. Biol. 9, 2914-2922 (2014).

25 Wang, M. \& Boddy, C. N. Examining the role of hydrogen bonding interactions in the substrate specificity for the loading step of polyketide synthase thioesterase domains. Biochemistry 47, 11793-11803 (2008).

26 Horsman, M. E., Hari, T. P. \& Boddy, C. N. Polyketide synthase and non-ribosomal peptide synthetase thioesterase selectivity: logic gate or a victim of fate? Nat. Prod. Rep. 33, 183-202 (2016).

$27 \mathrm{Li}, \mathrm{Y}$. et al. Functional Characterization of a Dehydratase Domain from the Pikromycin Polyketide Synthase. J. Am. Chem. Soc. 137, 7003-7006 (2015).

28 Zheng, J., Gay, D. C., Demeler, B., White, M. A. \& Keatinge-Clay, A. T. Divergence of multimodular polyketide synthases revealed by a didomain structure. Nat. Chem. Biol. 8, 615-621 (2012)

Supplementary Information accompanies the paper on The Journal of Antibiotics website (http://www.nature.com/ja) 